Result(s)* 142 patients were identified among 12 participating centers performing SLN mapping. In $64.8 \%$ of cases a lowvolume disease $(\leq 2 \mathrm{~mm})$ was found in SLNs: $33(23.2 \%)$ ITCs and 59 (41.6\%) micrometastases. Factors influencing volume of nodal metastases were: grading [p:0.002] (G1 associated with low-volume disease), LVSI [p:0.007] and MI >50\% [p:0.008] (both associated with macrometastases). There were: 20 (14.1\%) low-risk, 14 (9.8\%) intermediate, 88 (62\%) highintermediate and 20 (14.1\%) high-risk according to 2020ESGO/ESTRO/ESP risk group (on uterus). 17 (18.5\%) patients with low-volume disease (8 micrometastases and 9 ITCs) did not receive any adjuvant therapy. At a mean follow-up of 34.6 months (range 1 -215) months, 21 (14.8\%) relapses were recorded, only one among patients not receiving any adjuvant, none in the ESGO/ESTRO/ESP low risk group. The RFS at 2-years for the micrometastatic patients was 91\%, similar to ITCs patients (79.1\%), regardless of adjuvant treatment, but statistically better than patients with macrometastases (72.3\%) [p: 0.026]. There was a trend to distinct RFS according to ESGO/ESTRO/ESP risk group, but none of the comparisons reached significance. The only factors affecting RFS were deep MI [p:0.03] and cervical stromal invasion [p:0.046]. Conclusion* More than half of patients with positive SLNs had low-volume disease. Grading, MI and LVSI predicted volume of nodal metastases. MI and cervical invasion affected RFS; while adjuvant treatment did not seem significantly associated with RFS in patients with low-volume disease. Longer follow-up time and a larger sample size are needed to understand the role of adjuvant therapy in low-volume metastatic SLNs.

\section{REAL-WORLD OUTCOMES OF PATIENTS WITH ADVANCED ENDOMETRIAL CANCER: A RETROSPECTIVE COHORT STUDY OF US ELECTRONIC HEALTH RECORDS}

${ }^{1} S$ Banerjee*, ${ }^{2} \mathrm{G}$ Smith, ${ }^{1} \mathrm{~J}$ Lima, ${ }^{3} \mathrm{G}$ Long, ${ }^{3} \mathrm{~N}$ Alam, ${ }^{3} \mathrm{H}$ Nakamura, ${ }^{3} \mathrm{D}$ Meulendijks, ${ }^{2}$ BJ Monk. 'The Royal Marsden NHS Foundation Trust and Institute of Cancer Research, London, UK; ${ }^{2}$ University of Arizona College of Medicine, Creighton University School of Medicine at St Joseph's Hospital, Phoenix, USA; ${ }^{3}$ AstraZeneca Pharmaceuticals LP, Cambridge, UK

\subsection{6/ijgc-2021-ESG0.130}

Introduction/Background* Endometrial cancer (EC) is the most common gynaecological malignancy in the USA, and mortality from advanced/metastatic EC (AEC) is increasing. EC constitutes a heterogeneous group of diseases with distinct histological subtypes. We characterized the prognosis in routine clinical practice of women with uterine serous carcinoma (USC), endometrioid carcinoma, clear-cell carcinoma (CCC), carcinosarcoma, and EC not otherwise specified.

Methodology This retrospective study utilized electronic health records from the US Flatiron database, comprising structured and unstructured data from community and academic centres.

Abstract 271 Table 1 Patient characteristics

\begin{tabular}{|c|c|c|c|c|c|c|c|}
\hline & & $\begin{array}{l}\text { All patients } \\
(\mathrm{N}=2202)\end{array}$ & $\begin{array}{l}\text { Uterine serous } \\
\text { carcinoma } \\
(n=551)\end{array}$ & $\begin{array}{l}\text { Endometrioid } \\
\text { carcinoma } \\
\text { (n=1317) }\end{array}$ & $\begin{array}{c}\text { Clear-cell } \\
\text { carcinoma } \\
(n=92)\end{array}$ & $\begin{array}{l}\text { Carcino- } \\
\text { sarcoma } \\
(n=30)\end{array}$ & $\begin{array}{c}\text { EC not otherwise } \\
\text { specified } \\
(n=212)\end{array}$ \\
\hline \multicolumn{2}{|c|}{$\begin{array}{l}\text { Median age at advanced diagnosis, years } \\
\text { (interquartile range) }\end{array}$} & $66.0(59.0-72.0)$ & $69.0(64.0-74.0)$ & $64.0(56.0-70.0)$ & $68.5(60.5-74.0)$ & $71.5(63.0-76.0)$ & $65.5(59.5-72.5)$ \\
\hline \multirow{6}{*}{$\begin{array}{l}\text { Race/ethnicity, } \\
\text { n (\%) }\end{array}$} & Asian & $48(2.2)$ & $10(1.8)$ & $32(2.4)$ & $1(1.1)$ & $1(3.3)$ & $4(1.9)$ \\
\hline & Black/African American & $318(14.4)$ & $145(26.3)$ & $124(9.4)$ & $16(17.4)$ & $3(10.0)$ & $30(14.2)$ \\
\hline & Hispanic/Latino & $3(0.1)$ & $0(0.0)$ & $2(0.2)$ & $1(1.1)$ & 0 & 0 \\
\hline & White & $1401(63.6)$ & $309(56.1)$ & $886(67.3)$ & $60(65.2)$ & $16(53.3)$ & $130(61.3)$ \\
\hline & Other & $233(10.6)$ & $49(8.9)$ & $142(10.8)$ & $10(10.9)$ & $7(23.3)$ & $25(11.8)$ \\
\hline & Unknown & $199(9.0)$ & $38(6.9)$ & $131(10.0)$ & $4(4.4)$ & $3(10.0)$ & $23(10.9)$ \\
\hline \multirow{4}{*}{$\begin{array}{l}\text { ECOG score at } \\
\text { advanced } \\
\text { diagnosis, } n(\%)\end{array}$} & 0 & $1125(51.1)$ & $275(49.9)$ & $681(51.7)$ & $51(55.4)$ & $11(36.7)$ & $107(50.5)$ \\
\hline & 1 & $539(24.5)$ & $136(24.7)$ & $333(25.3)$ & $16(17.4)$ & $10(33.3)$ & $44(20.8)$ \\
\hline & 2 & $126(5.7)$ & $27(4.9)$ & $77(5.9)$ & $9(9.8)$ & $3(10.0)$ & $10(4.7)$ \\
\hline & Unknown & $412(18.7)$ & $113(20.5)$ & $226(17.2)$ & $16(17.4)$ & $6(20.0)$ & $51(24.1)$ \\
\hline \multirow{5}{*}{$\begin{array}{l}\text { Stage at initial } \\
\text { diagnosis, } \mathrm{n}(\%)\end{array}$} & 1 & $407(18.5)$ & $82(14.9)$ & $290(22.0)$ & $8(8.7)$ & $3(10.0)$ & $24(11.3)$ \\
\hline & II & $66(3.0)$ & $18(3.3)$ & $39(3.0)$ & $6(6.5)$ & 0 & $3(1.4)$ \\
\hline & III & $1073(48.7)$ & $253(45.9)$ & $700(53.2)$ & $45(48.9)$ & $14(46.7)$ & $61(28.8)$ \\
\hline & IV & $557(25.3)$ & $185(33.6)$ & $239(18.1)$ & $31(33.7)$ & $11(36.7)$ & $91(42.9)$ \\
\hline & Unknown & $99(4.5)$ & $13(2.4)$ & $49(3.7)$ & $2(2.2)$ & $2(6.7)$ & $33(15.6)$ \\
\hline \multirow{2}{*}{$\begin{array}{l}\text { Health practice } \\
\text { setting, } n(\%)\end{array}$} & Academic & $380(17.3)$ & $113(20.5)$ & $212(16.1)$ & $20(21.7)$ & $3(10.0)$ & $32(15.1)$ \\
\hline & Community & $1822(82.7)$ & $438(79.5)$ & 1105 (83.9) & $72(78.3)$ & $27(90.0)$ & $180(84.9)$ \\
\hline
\end{tabular}


Abstract 271 Table 2 Median OS and TFST from initiation of system treatment for recurrent/metastatic disease after advanced diagnosis

\begin{tabular}{|c|c|c|c|c|c|c|c|}
\hline & & $\begin{array}{l}\text { All patients } \\
\left(\mathrm{N}=2169^{*}\right)\end{array}$ & $\begin{array}{c}\text { Uterine } \\
\text { serous } \\
\text { carcinoma } \\
(n=536)\end{array}$ & $\begin{array}{l}\text { Endometrioid } \\
\text { carcinoma } \\
\qquad(n=1307)\end{array}$ & $\begin{array}{c}\text { Clear-cell } \\
\text { carcinoma } \\
(n=87)\end{array}$ & $\begin{array}{l}\text { Carcino- } \\
\text { sarcoma } \\
(n=29)\end{array}$ & $\begin{array}{c}\text { EC not } \\
\text { otherwise } \\
\text { specified } \\
(n=210)\end{array}$ \\
\hline \multirow{6}{*}{ os $^{\dagger}$} & Median OS $(95 \% \mathrm{Cl})$, months & $49.6(43.9-52.0)$ & $31.3(27.7-34.3)$ & $70.8(60.5-83.2)$ & $29.2(18.3-57.0)$ & $14.4(7.9-53.1)$ & $40.7(21.4-48.1)$ \\
\hline & Interquartile range for median OS, months & $18.1-89.5$ & $15.4-61.8$ & 28.4-NE & 16.1-NE & $6.8-53.1$ & 8.2-NE \\
\hline & Patients with event, $\mathrm{n}$ & 798 & 254 & 394 & 38 & 16 & 96 \\
\hline & Censored patients, $\mathrm{n}$ & 1371 & 282 & 913 & 49 & 13 & 114 \\
\hline & Median follow-up $(95 \% \mathrm{Cl})$, months & $33.0(30.6-35.0)$ & $32.2(26.1-36.3)$ & $33.7(31.0-35.6)$ & $30.4(22.2-33.9)$ & $24.1(12.0-33.0)$ & $35.6(26.8-43.3)$ \\
\hline & Interquartile range for median follow-up, months & $14.9-52.0$ & $14.5-51.6$ & 24.8-NE & 16.1-NE & $12.0-33.0$ & 8.2-NE \\
\hline \multirow{5}{*}{ TFST $^{\dagger}$} & Median TFST $(95 \% \mathrm{Cl})$, months & $13.6(12.1-15.0)$ & $10.6(9.6-12.3)$ & $18.9(15.8-21.7)$ & $10.8(7.7-16.3)$ & $9.6(6.7-20.2)$ & $8.3(6.0-10.7)$ \\
\hline & Patients with event, $\mathrm{n}$ & 1297 & 381 & 697 & 60 & 19 & 140 \\
\hline & Censored patients, $\mathrm{n}$ & 872 & 155 & 610 & 27 & 10 & 70 \\
\hline & Median follow-up ( $95 \% \mathrm{Cl})$, months & $34.0(31.2-36.7)$ & $37.8(34.4-40.6)$ & $31.7(29.5-35.0)$ & $30.6(22.2-51.4)$ & $25.7(12.0-86.8)$ & $42.3(31.7-45.7)$ \\
\hline & Interquartile range for median follow-up, months & $15.4-52.4$ & $16.5-57.2$ & $14.5-51.8$ & $21.4-52.8$ & $12.0-86.8$ & $19.2-58.9$ \\
\hline
\end{tabular}

*Thirty-three patients did not have recorded therapy following AEC diagnosis: 15 with serous-cell carcinoma; 10 with endometrioid carcinoma; five with clear-cell carcinoma; one with carcinosarcoma; and two with endometrial cancer not otherwise specified; ${ }^{\dagger}$ Crude Kaplan-Meier analyses (no adjustment for confounding factors). NE, not estimable

Women aged $\geq 18$ years diagnosed with AEC (initial diagnosis: stage III/IV, or early stage with subsequent locoregional/distal recurrence) between 1 January 2013 and 30 September 2020, inclusive, were eligible provided they received platinum-based chemotherapy at any time following diagnosis and had $\geq 2$ clinical visits. Patients were followed up from initiation of systemic treatment for recurrent/metastatic disease after advanced diagnosis until 31 March 2021, last available follow-up, or death (whichever occurred first). Overall survival (OS) and time to first subsequent therapy or death (TFST) were estimated with Kaplan-Meier methodology.

Result(s)* Overall, 2202 women with AEC were included; most $(82.7 \%)$ were treated in a community setting and pre-

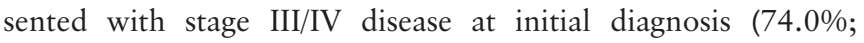
table 1). Compared with other subtypes, a higher proportion (26.3\%) of women with USC were Black/African American. Thirty-three $(1.5 \%)$ patients did not have recorded therapy following AEC diagnosis. The most common first systemic treatments for recurrent/metastatic disease were platinumbased combination chemotherapy (82.0\%), platinum-based single-agent chemotherapy (7.9\%), and platinum-based chemotherapy plus HER2-targeted therapy (2.9\%); median (interquartile range) duration was 9.2 (4.1-23.2) months. Median (95\% confidence interval [CI]) OS from initiation of first systemic treatment was shorter in patients with USC (31.3 [27.7-34.3] months), CCC (29.2 [18.3-57.0] months), and carcinosarcoma (14.4 [7.9-53.1] months) versus the overall population (49.6 [43.9-52.0] months; table 2), as was median TFST from initiation of first systemic treatment (table 2).

Conclusion* In this large real-world study, patients with advanced USC, CCC, and carcinosarcoma had poorer survival outcomes than the overall AEC population, demonstrating an unmet need in these populations, such as the requirement for more effective therapies.

\section{DOSTARLIMAB IN ADVANCED/RECURRENT MISMATCH REPAIR DEFICIENT/MICROSATELLITE INSTABILITY HIGH OR PROFICIENT/STABLE ENDOMETRIAL CANCER: THE GARNET STUDY}

${ }^{1} \mathrm{~A}$ Oaknin*, ${ }^{2} \mathrm{~L}$ Gilbert, ${ }^{3} \mathrm{AV}$ Tinker, ${ }^{4} \mathrm{~J}$ Brown, ${ }^{5} \mathrm{C}$ Mathews, ${ }^{6} \mathrm{~J}$ Press, ${ }^{7} \mathrm{R}$ Sabatier, ${ }^{8} \mathrm{DM}$ O'malley, ${ }^{9} \mathrm{~V}$ Samouëlian, ${ }^{10} \mathrm{~V}$ Boni, ${ }^{11} \mathrm{~L}$ Duska, ${ }^{12} \mathrm{~S}$ Ghamande, ${ }^{13} \mathrm{P}$ Ghatage, ${ }^{14} \mathrm{R}$ Kristeleit, ${ }^{15} \mathrm{C}$ Leath, ${ }^{16} \mathrm{~J}$ Veneris, ${ }^{16} \mathrm{~T}$ Duan, ${ }^{16} \mathrm{E} \mathrm{Im},{ }^{17} \mathrm{~B}$ Pothuri. ${ }^{1}$ Vall $\mathrm{d}^{\prime}$ Hebron University Hospital, Vall d'Hebron Institute of Oncology (VHIO), Barcelona, Spain; ${ }^{2}$ McGill University Health Centre Research Institute, Montreal, Quebec, Canada; ${ }^{3} B C$ Cancer, Vancouver, British Columbia, Canada; ${ }^{4}$ Division of Gynecologic Oncology, Levine Cancer Institute, Atrium Health, Charlotte, NC, USA; ${ }^{5}$ Women and Infants Hospital of Rhode Island, Providence, RI, USA; ${ }^{6}$ Swedish Cancer Institute Gynecologic Oncology and Pelvic Surgery, Seattle, WA, USA; ${ }^{7}$ Department of Medical Oncology, Institut Paoli Calmettes, Aix-Marseille University, Marseille, France; ${ }^{8}$ The Ohio State University - James Comprehensive Cancer Center, Columbus, OH, USA; ${ }^{9}$ Gynecologic Oncology Service, Department of Obstetrics and Gynecology, CHUM, Université de Montréal, Montreal, Quebec, Canada; ${ }^{10}$ START MadridCIOCC, Centro Integral Oncológico Clara Campal, Hospital Universitario HM Sanchinarro, Madrid, Spain; ${ }^{11}$ Emily Couric Clinical Cancer Center, University of Virginia, Charlottesville, VA, USA; ${ }^{12}$ Georgia Cancer Center, Augusta University, Augusta, GA, USA; ${ }^{13}$ Department of Gynecological Oncology, University of Calgary, Calgary, Alberta, Canada; ${ }^{14}$ Guy's and St Thomas' Hospital NHS Foundation Trust, London, UK; ${ }^{15} \mathrm{O}^{\prime} \mathrm{Neal}$ Comprehensive Cancer Center, University of Alabama at Birmingham, Birmingham, AL, USA; ${ }^{16}{ }^{\text {GlaxoSmithKline, }}$ Waltham, MA, USA; ${ }^{17}$ New York University, Department of Obstetrics and Gynecology, NYU Langone Health, Perlmutter Cancer Center, New York, NY, USA

\subsection{6/ijgc-2021-ESG0.131}

Introduction/Background* Dostarlimab is a humanised programmed death (PD-1) receptor monoclonal antibody approved for patients with mismatch mutation repair-deficient/microsatellite instability-high (dMMR/MSI-H) recurrent or advanced endometrial cancer (EC) that progressed on or after a platinum-based chemotherapy regimen. GARNET is a phase 1 study assessing the antitumour activity and safety of dostarlimab monotherapy in patients with advanced solid tumours. 